\title{
ACUMULACIÓN DE MATERIA SECA DEL CULTIVO DE PEPINO (Cucumis sativus L.) EN INVERNADERO
}

\section{DRY MATTER ACCUMULATION OF THE GREENHOUSE CUCUMBER CROP}

\author{
Fernando V. Barraza A. ${ }^{1}$ \\ Recibido para publicación: Septiembre 19 de 2011 - Aceptado para publicación: Agosto 1 de 2012
}

\begin{abstract}
RESUMEN
El estudio de la fisiología del crecimiento de los cultivos hortícolas bajo la modalidad de agricultura intensiva protegida en invernaderos es importante en la generación de información sobre algunos aspectos que permitan mejorar el manejo agronómico, por ejemplo en lo referente a nutrición mineral. Por la importancia del pepino en este contexto, se cultivó en los invernaderos de la Universidad Autónoma Chapingo (México) el híbrido "Saber" con cuatro concentraciones de la solución nutritiva universal de Steiner (25, 75, 125 y 175\%), bajo un diseño experimental de bloques al azar con tres repeticiones, en el periodo comprendido entre abril y agosto de 2006. Durante 135 días después del trasplante, se cuantificó cada 15 días la materia seca de tallos, hojas, zarcillos, flores, frutos, total y el rendimiento. Con la solución nutritiva al 175\% se obtuvo mejor expresión del crecimiento a través de mayor acumulación de materia seca y rendimiento de $8,20 \mathrm{~kg}$ por planta, que superó en 59,22 y $15 \%$ a lo obtenido con las concentraciones del 25,75 y $125 \%$, respectivamente, lo que muestra la respuesta del cultivo de pepino al manejo diferencial del suministro de nutrimentos, ya que con la solución nutritiva al $25 \%$ de concentración se presentaron valores más bajos de rendimiento y de acumulación de materia seca total y de órganos de la planta.
\end{abstract}

Palabras clave: Cucumis sativus L., análisis de crecimiento, modelo logístico, solución nutritiva, invernadero.

\begin{abstract}
Horticultural cultivations growth physiology under the greenhouse intensive protected agriculture modality study is important in the generation of information on some aspects that allow to improve the agronomic handling, for example mineral nutrition regarding. For the importance of the cucumber crop in this context, it was cultivated in greenhouse at the Universidad Autónoma Chapingo (México) the hybrid cucumber "Saber" with four concentrations of the Steiner's universal nutritious solution (25, 75, 125 and 175\%), under the random blocks experimental design, with three repetitions, between April and August 2006. During 135 days after the transplant, every 15 days, it was quantified the dry matter of stems, leaves, trendrils, flowers fruits,
\end{abstract}

'Doctor en Ciencias en Horticultura, profesor investigador Universidad de Córdoba, Facultad de Ciencias Agrícolas, Grupo Agricultura Sostenible, Carrera 6 No.76-103, Montería, Colombia. Fax: 7860255. E-mail: fbarraza@correo.unicordoba.edu.co 
whole dry matter and yield. With the nutritious solution to $175 \%$ it was obtained highest expression of crop growth through bigger accumulation of dry matter and yield of $8.20 \mathrm{~kg}$ by plant, that overcame in 59, 22 and $15 \%$ to the yield obtained with the concentrations of the 25,75 and $125 \%$, respectively, this shows the cucumber cultivation response to the differential nutrients supply handling, because with the nutrient solution to $25 \%$ lower values of yield, whole dry matter and organs accumulation were obtained.

Key words: Cucumis sativus L., growth analysis, logistic model, nutritious solution, greenhouse.

\section{INTRODUCCIÓN}

México es el segundo exportador mundial de pepino y el primer proveedor del mercado de Estados unidos, principalmente en la época de invierno, cuando se produce esta hortaliza en invernaderos y se aumenta significativamente el rendimiento, desde 25-50 t $\cdot$ ha $^{-1}$ (campo abierto) hasta 80-180 th ha-1 ${ }^{-1}$ en vista principalmente del control computarizado del clima (para evitar las fluctuaciones que en el caso de siembras a campo abierto ocasionan gran consumo de energía en las plantas y por ende, retraso en la producción de materia seca), mejoramiento en las técnicas de producción e introducción de sustratos artificiales y genotipos de alta productividad (Intagri 2012).

La modalidad de cultivo intensivo de hortalizas bajo invernadero en México sobrepasa las 12000 ha, con un valor de inversión de más de 3000 millones de dólares (Intagri 2012). Ello ha generado un cambio sustancial en la manera de analizar los diferentes factores que intervienen en la producción. Bajo este contexto, se ha identificado al pepino como uno de los cultivos que muestra mayor flexibilidad para adaptarse a los cambios en distintos sistemas de producción, entre los que cabe destacar la respuesta del rendimiento ante el suministro diferencial de nutrimentos (Randolph 1999), lo que sirve de punto de partida para direccionar investigaciones sobre el comportamiento fisiológico del cultivo a través del análisis de crecimiento en diferentes condiciones nutrimentales.

El crecimiento vegetal y la consecuente acumulación de materia seca están relacionados directamente con la absorción continua de nutrimentos minerales, la cual se produce solamente si aumenta el tamaño de la planta. En caso contrario, especialmente para las hortalizas, caracterizadas por su periodo vegetativo relativamente corto, se deja de aprovechar el potencial productivo de los genotipos de alto rendimiento, disponibles y accesibles en la actualidad y por lo tanto, se obtienen rendimientos por debajo de una buena redituabilidad económica, con la consiguiente pérdida de competitividad y sostenibilidad (Güenkov 1974; Valadez 1998).

Lo anterior favorece para el sistema de producción de pepino un mercado en expansión y más consolidado, si se tiene en cuenta que el $70 \%$ del consumo mundial de esta hortaliza lo comparten con los Estados Unidos otros países como China, Irán, Turquía y Japón, cuya demanda de 12,4 millones de toneladas $(52,4 \%$ del consumo mundial) se satisface a través de importaciones, ya que su producción interna no alcanza a cubrir dicho requerimiento (Barreiro 1999). 
Con el estudio de la relación fisiológica fuente-demanda del cultivo de pepino en invernadero, Marcelis (1992) ha demostrado que la materia seca que se distribuye hacia los frutos representa hasta $60 \%$ del total de materia seca producida por fotosíntesis, y reporta una correlación positiva entre el número de frutos por planta y el crecimiento de las partes vegetativas, lo que corrobora la importancia de realizar y profundizar investigaciones sobre la fisiología de la producción de materia seca, ya que de su mayor acumulación a través del tiempo depende la obtención de los más altos rendimientos (Tanaka y Yamaguchi 1984) y se genera información que ayuda a generar recomendaciones para que los agricultores hagan uso más eficiente de los recursos y la tecnología, como por ejemplo el empleo de variedades e híbridos ginoicos de alto rendimiento, fertirrigación, métodos de protección de cultivos y de preservación de la biodiversidad (Nátr y Lawlor 2005).

La aproximación más precisa que hacen los investigadores al análisis y cuantificación de los factores que influyen en el rendimiento y desarrollo vegetal mediante operaciones y modelos matemáticos para ambientes particulares, es el estudio de la acumulación neta de fotosintatos a través del tiempo, denominada análisis de crecimiento (Clavijo 1989; Gardner et al. 1990; Nátr y Lawlor 2005). Para Ilevarlo a cabo, generalmente se hacen mediciones de materia seca a intervalos frecuentes. Otra información en el análisis de crecimiento, como por ejemplo los índices de eficiencia fisiológica del cultivo, es obtenida mediante cálculos matemáticos. En la actualidad la utilización de ordenadores rápidos y programas de cómputo que permiten el manejo de grandes cantidades de datos han facilitado considerablemente estos procesos (Calvo et al. 1994; Gardner et al. 1990; Hunt 1990).

El análisis de crecimiento presenta dos enfoques en su realización. El primero es el clásico, que implica mediciones hechas de manera precisa a intervalos continuos (1-2 semanas) sobre un número relativamente amplio de plantas. El segundo, conocido como funcional, implica mediciones a intervalos más frecuentes (2-3 días) sobre un número pequeño de plantas. Ambos enfoques proporcionan valores promedios de los cambios cuantitativos que ocurren a través de cualquier intervalo de tiempo en particular (por ejemplo longitud y diámetro del tallo, número de hojas, área foliar) los cuales tienen gran importancia para los agrónomos al analizar la comunidad de plantas de manera integral, debido a que ello representa el rendimiento económico que resulta de la acumulación neta de $\mathrm{CO}_{2}$ asimilado durante todo el ciclo de crecimiento (Gardner et al. 1990).

Para hacer un mejor uso de los materiales y tiempo del investigador, está más extendido el uso del análisis de crecimiento funcional, que además facilita la elaboración de modelos del crecimiento con alta confiabilidad (Gardner et al. 1990; Hunt 1982) y la realización de curvas que caractericen el comportamiento de las variables del crecimiento con datos ajustados, obtenidos mediante variedad de modelos matemáticos no lineales entre los que sobresalen el logístico, Gompertz, Weibull y Richards, exponencial, MichaelisMenten y monomolecular (Rojas et al. 2008) y procedimientos como la regresión lineal, cuadrática y logarítmica, derivada y desarrollo 
de modelos polinomiales, que han sido trabajados ampliamente en cultivos (Alonso et al. 2003; Calvo et al. 1994; Charles-Edwards et al. 1986; Evans 1972; Gardner et al. 1990; Hunt y Parsons 1974; Hunt 1978, 1982, 1990; Jean 1984; Krug 1985).

Dada la importancia del análisis de crecimiento en los cultivos hortícolas y que en México el cultivo de pepino en invernadero es un sistema de producción cada vez más en expansión por las crecientes posibilidades de exportación, además de que no se tienen estudios básicos sobre aspectos de la fisiología de su crecimiento, se hizo la presente investigación con el objetivo de cuantificar, bajo diferentes concentraciones nutrimentales, la acumulación de materia seca total y por órganos en el cultivo del pepino, así como caracterizar su evolución en el tiempo y su efecto en el rendimiento.

\section{MATERIALES Y MÉTODOS}

La investigación se llevó a cabo entre el 14 de abril y el 14 de agosto de 2006 en los invernaderos del campo experimental de la Universidad Autónoma Chapingo, México, ubicada a $19^{\circ} 29^{\prime}$ de Latitud Norte y $98^{\circ} 53^{\prime}$ de Longitud Oeste, a 2250 m de altitud. Se sembró el híbrido de pepino Saber ${ }^{\circledR}$. Se obtuvieron plántulas mediante la siembra de una semilla por cavidad en charolas de unisel de 200 cavidades, con sustrato de turba (Peat-Moss ${ }^{\circledR}$ ). A los 25 días después de la siembra, se realizó el trasplante, cuando las plántulas tuvieron la primera hoja verdadera completamente expandida. La unidad experimental consistió en una planta de pepino trasplantada en una bolsa de polietileno negro, de 0,06 $\mathrm{m}^{3}$ de volumen. El sustrato fue tezontle rojo. La separación entre hileras de plantas fue de 1,0 m, y de 0,30 m entre plantas. Se utilizó un diseño experimental en bloques al azar con cuatro tratamientos y tres repeticiones, en una superficie de $99 \mathrm{~m}^{-2}$, con un total de 156 plantas. Los tratamientos (Tn) correspondieron a concentraciones de la solución nutritiva universal de Steiner (Steiner 1961): $25 \%$ (T1), 75\% (T2), 125\% (T3) y $175 \%$ (T4), cuya composición de nutrimentos (meq $\left.\mathrm{L}^{-1}\right)$ para una concentración del 100\% fue: $\mathrm{NO}_{3}{ }^{-}, 12 ; \mathrm{H}_{2} \mathrm{PO}_{4}^{-}, 1 ; \mathrm{SO}_{4}^{-}, 7 ; \mathrm{Ca}^{++}, 9 ; \mathrm{Mg}^{++}, 4$ $\mathrm{y} \mathrm{K}^{+}, 7$. Durante 30 días después del trasplante (ddt), de acuerdo con lo indicado por Castro y Pérez (2005) se aplicaron según cada tratamiento, $150 \mathrm{~mL}$ diarios de la respectiva solución nutritiva por planta, distribuidos en tres riegos de $50 \mathrm{~mL}$; de los 31 a los 60 ddt se aplicaron $300 \mathrm{~mL}$ diarios por planta, en tres riegos de $100 \mathrm{~mL}$; del día 61 al 135 después del trasplante, se aplicaron $600 \mathrm{~mL}$ diarios por planta distribuidos en tres riegos de 200 $\mathrm{mL}$. El intervalo entre riegos fue de cuatro horas. Se utilizó sistema hidropónico abierto con riego por goteo. El cultivo se desarrolló a libre crecimiento, sin realizar podas, y se hizo tutorado de espaldera a los 15 ddt mediante malla plástica con cuadrícula de $10 \mathrm{~cm}$. Se hicieron aplicaciones preventivas cada 15 días de oxicloruro de cobre con mancozeb, en concentración de 0,5 $\mathrm{g} \mathrm{L}^{-1}$, y cuando fue necesario malathion para el control de plagas, en concentración de $0,5 \mathrm{~mL} \mathrm{~L}^{-1}$.

Desdeel trasplantehasta los 135 díasposteriores, se realizaron muestreos destructivos cada 15 días, en los que se registraron datos de peso de materia seca de tallos, hojas, zarcillos, flores, frutos y peso de materia seca total sin incluir raíz, todos expresados en gramos. Se tomó el promedio a tres plantas seleccionadas al azar 
en los tres surcos centrales de cada repetición. Para la obtención del peso seco, las muestras vegetales se secaron en horno Blue- $\mathrm{M}^{\circledR}$ POM$326-\mathrm{F}$, a $65^{\circ} \mathrm{C}$ hasta la obtención de peso constante y se pesaron en balanza digital Sartorius 1205 MP $^{\circledR}$. Se tomaron además datos de rendimiento total por planta durante la etapa de fructificación del cultivo, expresado en g por planta. Se tomó el promedio de tres plantas seleccionadas al azar y marcadas en los tres surcos centrales de cada repetición.

Para determinar el comportamiento inicial de los datos de las variables estudiadas, se utilizó el software Curve Expert versión 1.3 (Hyams 2003). Por las características de la información obtenida se aplicó el procedimiento de regresión no lineal mediante el software SAS 9.0 (SAS Institute Inc. 2002) con el modelo logístico:

$$
y=\frac{A}{\left(1+B \cdot e^{-C \cdot x}\right)}
$$

donde:

$y$ : variable respuesta (materia seca, en función de $x$ )

$\mathrm{x}$ : tiempo (días después del trasplante)

A: asíntota, valor de y cuando $x$ tiende a infinito $B \cdot e^{-c x}$ factor de amortiguamiento, donde:

B: corresponde a un factor de amplitud, no tiene significado biológico y solo toma lugar en el tiempo inicial, cuando $x=0$

C: factor de estabilidad relacionado con el valor de $\mathrm{x}$

e: número Euler

Para el análisis estadístico de las variables en estudio se realizó análisis de varianza (ANAVA) y prueba de comparación de medias de Tukey
( $\alpha \leq 0.05)$ mediante el software SAS 9.0 (SAS Institute Inc. 2002).

\section{RESULTADOS Y DISCUSIÓN}

Los modelos logísticos obtenidos para materia seca de tallos, hojas, zarcillos y total se presentan en la Tabla 1. Su comportamiento a través del tiempo fue sigmoide (Figura 1). Para cultivo de pepino, similares resultados fueron encontrados por Marcelis (1992). La trayectoria observada en la Figura 1 explica el crecimiento de numerosas plantas anuales a través del tiempo y presenta tres fases principales que son: logarítmica o exponencial, lineal y de senescencia (Barraza 2001; Fogg 1967; Gardner et al. 1990; Pérez y Martínez 1994; Salisbury y Ross 1994; Wild 1992). De acuerdo con los modelos logísticos obtenidos (Tabla 1) se encontró que la correlación entre datos observados y estimados es cercana a 1, lo que indica confiabilidad de los modelos para estimar la acumulación materia seca.

La materia seca de flores y frutos no presentó trayectoria sigmoide a través del tiempo y no se ajustó al modelo logístico. Similares resultados en este sentido fueron encontrados para cultivo de melón por Barraza (2005). Es posible que ello guarde relación con la caída de flores y frutos que ocurre en dichas especies cucurbitáceas de manera natural, y que según Peil y Galvez (2005) puede ser hasta de un $70 \%$, y puede ser mayor en la medida que el suministro de nutrimentos disminuye, ya que bajo dichas condiciones se puede presentar menor actividad meristemática en la planta, que trae como consecuencia que no aparezcan nuevas flores, o que las mismas no dieran origen a frutos, por la poca fuerza de la fuente 
Tabla 1. Modelos logísticos para estimar acumulación de materia seca de tallos, hojas, zarcillos y total de la planta de pepino, en cuatro concentraciones de la solución nutritiva de Steiner. Chapingo, México, 2006.

\begin{tabular}{|c|c|c|}
\hline \multicolumn{3}{|c|}{ Materia seca de tallos } \\
\hline $25 \%$ & $y=15,46 /\left(1+57,3793 \mathrm{e}^{-0,0637^{*} d d t}\right)$ & 0,98 \\
\hline $75 \%$ & $y=26,36 /\left(1+27,6516 \mathrm{e}^{-0,0476^{*} d d t}\right)$ & 0,96 \\
\hline $125 \%$ & $y=31,44 /\left(1+26,3684 \mathrm{e}^{-0,0437^{*} d d t}\right)$ & 0,97 \\
\hline $175 \%$ & $y=23,40 /\left(1+51,2402 \mathrm{e}^{-0,0765^{*} d d t}\right)$ & 0,94 \\
\hline \multicolumn{3}{|c|}{ Materia seca de hojas } \\
\hline $25 \%$ & $y=42,41 /\left(1+584,7 \mathrm{e}^{-0,1236^{*} d d t}\right)$ & 0,96 \\
\hline $75 \%$ & $y=70,52 /\left(1+23,8 \mathrm{e}^{-0,0478^{*} d d t}\right)$ & 0,96 \\
\hline $125 \%$ & $y=77,98 /\left(1+31,6 e^{-0,0548^{*} d d t}\right)$ & 0,95 \\
\hline $175 \%$ & $y=81,98 /\left(1+19,9 e^{-0,0473^{*} d d t}\right)$ & 0,95 \\
\hline \multicolumn{3}{|c|}{ Materia seca de zarcillos } \\
\hline $25 \%$ & $y=1,61 /\left(1+177,5 \mathrm{e}^{-0,0859^{*} d d t}\right)$ & 0,94 \\
\hline $75 \%$ & $y=2,42 /\left(1+57,0 e^{-0,0859 * d d t}\right)$ & 0,96 \\
\hline $125 \%$ & $y=2,63 /\left(1+38,8 e^{-0,0600^{*} d d t}\right)$ & 0,96 \\
\hline $175 \%$ & $y=2,36 /\left(1+155,0 e^{-0,0973^{*} d d t}\right)$ & 0,95 \\
\hline \multicolumn{3}{|c|}{ Materia seca total } \\
\hline $25 \%$ & $y=231,366 /\left(1+97,1809 \mathrm{e}^{-0,054099^{*} d d t}\right)$ & 0,99 \\
\hline $75 \%$ & $y=467,455 /\left(1+112,358 \mathrm{e}^{-0,049863^{*} d d t}\right)$ & 0,99 \\
\hline $125 \%$ & $y=472,763 /\left(1+113,972 e^{-0,0522^{*} d d t}\right)$ & 0,99 \\
\hline $175 \%$ & $y=585,034 /\left(1+78,7891 e^{-0,044108^{*} d d t}\right)$ & 0,99 \\
\hline
\end{tabular}

y: materia seca; ddt: días después del trasplante; PV: proporción de variabilidad que indica la confiabilidad del modelo = suma de cuadrados de regresión/suma de cuadrados total.

capaz de sostenerla como consecuencia de un deficiente transporte de fotoasimilados, relacionado con la poca disponibilidad nutrimental (Liebig 1980).

En general la materia seca de tallos y hojas presentó una tendencia a disminuir en la medida que lo hicieron las concentraciones de la solución nutritiva. Para el caso de materia seca de tallos hubo la menor expresión con la concentración del 25\%, entre los 30 y 75 ddt y a los 135 ddt. Con la solución del 75\% hubo menores valores a los 30, 60 y 75 ddt; igual situación ocurrió con la solución del 125\% a los 60 y 75 ddt (Tabla 2).

En lo que respecta a materia seca de hojas, hubo menor acumulación a los 30 ddt con las soluciones al 25 y $75 \%$ y a los 45 y $105 \mathrm{ddt}$ con las soluciones al 25, 75 y 125\% (Tabla 2).

La materia seca de zarcillos prácticamente no fue afectada por la variación de las concentraciones nutrimentales. Solo a los 60 ddt tuvo su menor expresión en las concentraciones del 25 y 75\% (Tabla 2).

La menor expresión de materia seca de flores se presentó con la concentración del 25\% a los 30 y 45 ddt. Con el 75\% de concentración la menor expresión ocurrió a los 30, 45 y 120 ddt, mientras que con la concentración del 175\% la menor expresión se dio a los 30 y 120 ddt (Tabla 3). Los periodos en los que se 

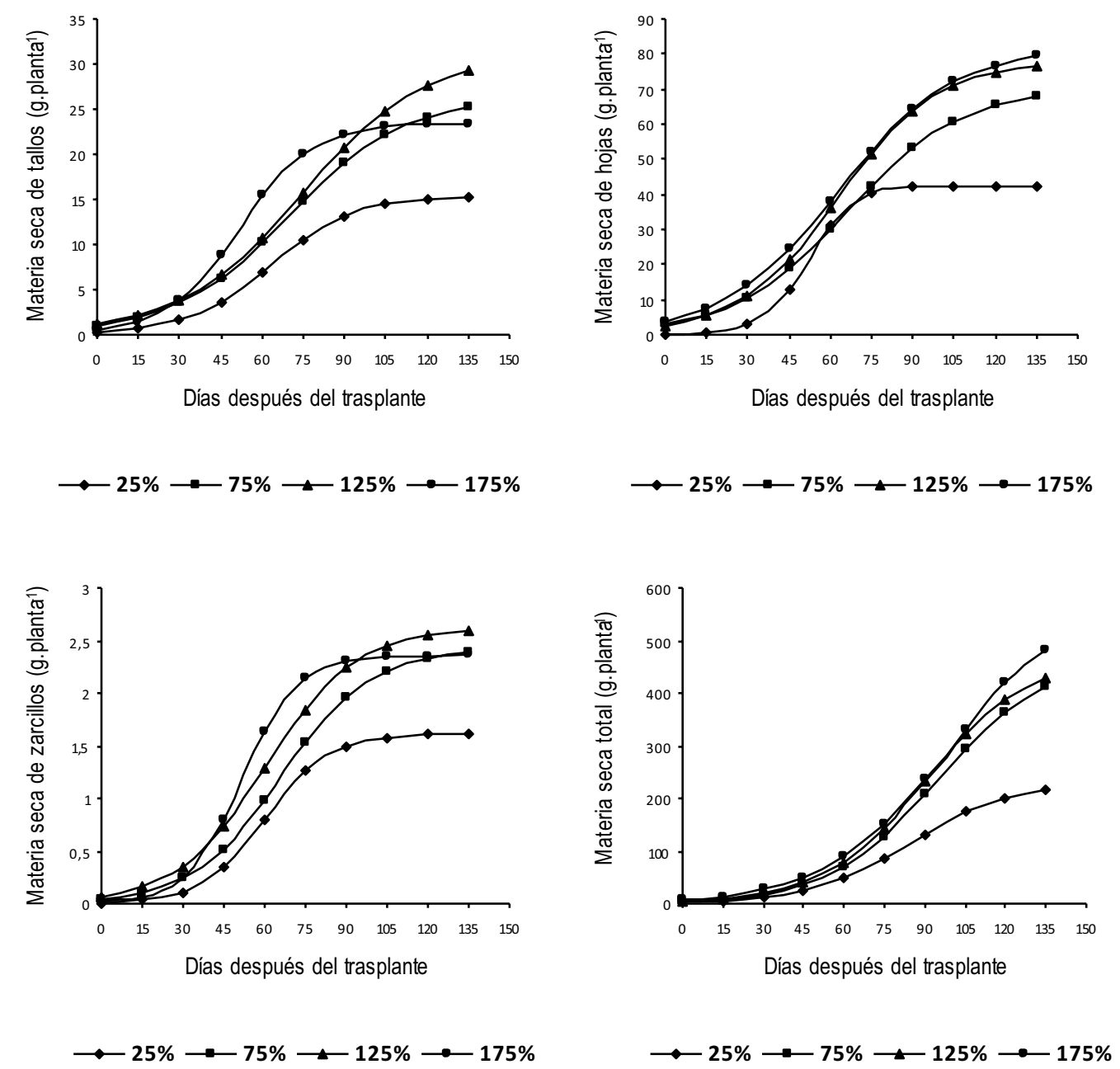

Figura 1. Acumulación de materia seca de tallos, hojas, zarcillos y total del cultivo de pepino, en cuatro concentraciones $(25 \%, 75 \%, 125 \%$ y $175 \%)$ de la solución nutritiva de Steiner, representada mediante modelos logísticos. Chapingo, México, 2006.

encontró menor materia seca de flores en las concentraciones del 25 y $75 \%$ posiblemente están relacionados con un alto porcentaje de aborto de flores, que conduce en lo posterior a un menor número de frutos, debido al reducido crecimiento general (De Lint y Heij 1980).

Respecto a la materia seca de frutos, con las soluciones nutritivas al 25,75 y $125 \%$ se dio menos acumulación a los 45 ddt. Para el caso de la solución al $25 \%$ se presentó también menor expresión a los 75, 120 y 135 ddt, con diferencias estadísticas significativas al resto de las soluciones nutritivas empleadas (Tabla 3).

En lo que concierne a materia seca total, con las concentraciones del 75, 125 y $175 \%$ se observó estadísticamente igual acumulación, con valores mayores respecto a la concentración del $25 \%$ desde los 30 ddt hasta el final del ciclo del cultivo, con diferencias estadísticas significativas con este último tratamiento, excepto para el periodo comprendido entre los 30 y 60 ddt para la solución al 75\% y a los 60 ddt para la solución al 125\% (Tabla 3). 
Tabla 2. Comparación de medias de materia seca de tallos, hojas y zarcillos de la planta de pepino, en cuatro concentraciones de la solución nutritiva de Steiner. Chapingo, México, 2006.

\begin{tabular}{|c|c|c|c|c|c|}
\hline DDT & $25 \%$ & $75 \%$ & $125 \%$ & $175 \%$ & DMS \\
\hline \multicolumn{6}{|c|}{ Materia seca de tallos } \\
\hline 0 & $0,02^{z} \mathrm{a}$ & $0,02 \mathrm{a}$ & $0,02 \mathrm{a}$ & $0,02 \mathrm{a}$ & 0,008 \\
\hline 15 & $0,08 \mathrm{a}$ & $0,07 \mathrm{a}$ & $0,08 \mathrm{a}$ & $0,08 \mathrm{a}$ & 0,049 \\
\hline 30 & $0,97 \mathrm{~b}$ & $1,33 a b$ & $1,56 \mathrm{a}$ & $1,59 \mathrm{a}$ & 0,565 \\
\hline 45 & $3,57 \mathrm{~b}$ & $8,27 \mathrm{a}$ & $8,22 \mathrm{a}$ & $10,14 \mathrm{a}$ & 3,750 \\
\hline 60 & $8,40 \mathrm{~b}$ & $10,86 \mathrm{~b}$ & $13,67 \mathrm{ab}$ & $17,46 \mathrm{a}$ & 5,634 \\
\hline 75 & $9,05 \mathrm{~b}$ & $16,66 \mathrm{ab}$ & $15,95 \mathrm{ab}$ & $18,35 \mathrm{a}$ & 8,925 \\
\hline 90 & 13,09 a & $14,90 \mathrm{a}$ & $17,88 \mathrm{a}$ & $18,26 \mathrm{a}$ & 10,353 \\
\hline 105 & $14,36 \mathrm{a}$ & $25,10 \mathrm{a}$ & $25,37 \mathrm{a}$ & $24,00 \mathrm{a}$ & 13,243 \\
\hline 120 & $16,14 \mathrm{a}$ & $21,14 \mathrm{a}$ & $27,52 \mathrm{a}$ & $21,33 \mathrm{a}$ & 15,488 \\
\hline 135 & $14,62 \mathrm{~b}$ & $27,35 \mathrm{a}$ & 29,98 a & $28,21 \mathrm{a}$ & 7,223 \\
\hline \multicolumn{6}{|c|}{ Materia seca de hojas } \\
\hline 0 & $0,05 \mathrm{a}$ & $0,06 \mathrm{a}$ & 0,06 a & $0,05 \mathrm{a}$ & 0,015 \\
\hline 15 & $0,39 a$ & $0,42 \mathrm{a}$ & $0,45 \mathrm{a}$ & $0,41 \mathrm{a}$ & 0,175 \\
\hline 30 & $3,26 \mathrm{~b}$ & $4,16 \mathrm{ab}$ & $5,16 \mathrm{a}$ & $5,22 \mathrm{a}$ & 1,812 \\
\hline 45 & $12,01 \mathrm{~b}$ & $28,52 \mathrm{ab}$ & $26,13 \mathrm{ab}$ & $35,11 \mathrm{a}$ & 21,481 \\
\hline 60 & 33,7 a & $35,59 \mathrm{a}$ & $44,84 \mathrm{a}$ & 48,16 a & 15,866 \\
\hline 75 & $33,48 \mathrm{a}$ & $35,24 \mathrm{a}$ & $43,57 \mathrm{a}$ & $42,16 \mathrm{a}$ & 22,705 \\
\hline 90 & 51,79 a & 46,49 a & $57,68 \mathrm{a}$ & $60,04 \mathrm{a}$ & 39,055 \\
\hline 105 & $41,87 \mathrm{~b}$ & $67,44 \mathrm{ab}$ & $68,95 \mathrm{ab}$ & $75,51 \mathrm{a}$ & 30,917 \\
\hline 120 & $44,65 \mathrm{~b}$ & $71,72 \mathrm{ab}$ & 98,74 a & $71,90 \mathrm{ab}$ & 38,742 \\
\hline 135 & $35,94 \mathrm{c}$ & $62,27 \mathrm{~b}$ & $60,66 \mathrm{~b}$ & 84,88 a & 18,762 \\
\hline \multicolumn{6}{|c|}{ Materia seca de zarcillos } \\
\hline 30 & $0,04 \mathrm{a}$ & 0,07 a & $0,07 \mathrm{a}$ & $0,08 \mathrm{a}$ & 0,049 \\
\hline 45 & $0,34 \mathrm{a}$ & $0,78 \mathrm{a}$ & $0,96 \mathrm{a}$ & 0,83 a & 0,662 \\
\hline 60 & $0,94 \mathrm{~b}$ & $1,03 \mathrm{~b}$ & $1,46 a b$ & $1,83 \mathrm{a}$ & 0,679 \\
\hline 75 & $0,96 \mathrm{a}$ & $1,33 \mathrm{a}$ & $1,69 \mathrm{a}$ & $1,78 \mathrm{a}$ & 0,897 \\
\hline 90 & $1,77 \mathrm{a}$ & $1,83 \mathrm{a}$ & $2,04 \mathrm{a}$ & $2,16 \mathrm{a}$ & 1,340 \\
\hline 105 & $1,70 \mathrm{a}$ & $2,66 \mathrm{a}$ & $2,38 \mathrm{a}$ & $2,67 \mathrm{a}$ & 1,178 \\
\hline 120 & 1,49 a & $2,20 \mathrm{a}$ & $3,10 \mathrm{a}$ & $2,45 \mathrm{a}$ & 1,671 \\
\hline 135 & $1,52 \mathrm{a}$ & 2,26 a & $2,30 \mathrm{a}$ & $2,27 \mathrm{a}$ & 1,682 \\
\hline
\end{tabular}

${ }^{z}$ Valores con la misma letra en cada hilera son estadísticamente iguales (Tukey, $\left.\alpha \leq 0,05\right)$. DDT: días después del trasplante; DMS: diferencia mínima significativa.

Se observó que en términos generales, la acumulación de materia seca total fue mayor en la medida que aumentó la concentración de la solución nutritiva. Para el caso de la concentración de la solución al 25\%, es posible que se haya visto afectado el proceso fotosintético, en el cual participan en gran medida los nutrimentos extraídos del suelo o sustrato por parte del cultivo, lo que pudo propiciar que las plantas no expresaran de manera óptima su potencial productivo (Visser, 1980; Skierkowski y Dobrzanska, 1980, Challa y Hauvelink 1993).

De acuerdo con Grageda (1999), en cultivo de pepino, como hortaliza productora de frutos, 
Tabla 3. Comparación de medias de materia seca de flores, frutos y total de la planta de pepino, en cuatro concentraciones de la solución nutritiva de Steiner. Chapingo, México, 2006.

\begin{tabular}{|c|c|c|c|c|c|}
\hline DDT & $25 \%$ & $75 \%$ & $125 \%$ & $175 \%$ & DMS \\
\hline \multicolumn{6}{|c|}{ Materia seca de flores } \\
\hline 30 & $0,08^{z} b$ & $0,11 \mathrm{~b}$ & 0,19 a & $0,15 a b$ & 0,082 \\
\hline 45 & $0,58 \mathrm{~b}$ & $1,32 \mathrm{ab}$ & $1,48 \mathrm{a}$ & $1,86 \mathrm{a}$ & 0,767 \\
\hline 60 & $1,53 \mathrm{a}$ & $1,70 \mathrm{a}$ & $2,18 \mathrm{a}$ & $2,27 \mathrm{a}$ & 2,922 \\
\hline 75 & $1,28 \mathrm{a}$ & $1,85 \mathrm{a}$ & $1,91 \mathrm{a}$ & $1,88 \mathrm{a}$ & 1,742 \\
\hline 90 & $1,62 \mathrm{a}$ & $2,53 \mathrm{a}$ & $1,88 \mathrm{a}$ & $2,25 \mathrm{a}$ & 2,131 \\
\hline 105 & $1,38 \mathrm{a}$ & $1,61 \mathrm{a}$ & $1,87 \mathrm{a}$ & $2,01 \mathrm{a}$ & 1,182 \\
\hline 120 & $1,19 b$ & $2,54 a b$ & $3,91 \mathrm{a}$ & $3,16 \mathrm{ab}$ & 2,718 \\
\hline 135 & $1,34 \mathrm{a}$ & $2,45 \mathrm{a}$ & $1,73 \mathrm{a}$ & $2,33 \mathrm{a}$ & 1,709 \\
\hline \multicolumn{6}{|c|}{ Materia seca de frutos } \\
\hline 45 & $0,18 d$ & $1,58 \mathrm{c}$ & $7,24 \mathrm{~b}$ & 8,45 a & 1,123 \\
\hline 60 & $17,70 \mathrm{a}$ & 37,80 a & 20,50 a & 30,80 a & 29,21 \\
\hline 75 & $15,10 \mathrm{~b}$ & $57,70 \mathrm{a}$ & 60,90 a & 69,60 a & 16,05 \\
\hline 90 & $23,40 \mathrm{a}$ & $23,50 \mathrm{a}$ & $34,30 \mathrm{a}$ & $21,60 \mathrm{a}$ & 37,79 \\
\hline 105 & $6,70 \mathrm{a}$ & $25,10 \mathrm{a}$ & $28,60 \mathrm{a}$ & $31,00 \mathrm{a}$ & 24,65 \\
\hline 120 & $0,00 \mathrm{~b}$ & $48,60 \mathrm{a}$ & 30,00 a & $44,30 \mathrm{a}$ & 57,87 \\
\hline 135 & $3,48 \mathrm{~d}$ & $9,41 \mathrm{c}$ & $0,00 \mathrm{~b}$ & $16,20 \mathrm{a}$ & 18,23 \\
\hline \multicolumn{6}{|c|}{ Materia seca total } \\
\hline 0 & $0,06 \mathrm{a}$ & 0,07 a & $0,07 \mathrm{a}$ & $0,07 \mathrm{a}$ & 0,02 \\
\hline 15 & $0,47 \mathrm{a}$ & $0,49 \mathrm{a}$ & $0,52 \mathrm{a}$ & $0,49 \mathrm{a}$ & 0,22 \\
\hline 30 & $4,35 \mathrm{~b}$ & $5,66 \mathrm{ab}$ & $6,98 \mathrm{a}$ & $7,05 \mathrm{a}$ & 2,40 \\
\hline 45 & $16,68 \mathrm{~b}$ & $40,47 \mathrm{ab}$ & 44,03 a & 56,38 a & 24,19 \\
\hline 60 & $67,32 \mathrm{~b}$ & $94,83 a b$ & $95,38 a b$ & $113,31 \mathrm{a}$ & 28,99 \\
\hline 75 & $77,40 \mathrm{~b}$ & 139,47 a & 159,32 a & $170,27 \mathrm{a}$ & 39,56 \\
\hline 90 & $135,17 \mathrm{a}$ & 163,53 a & $197,20 \mathrm{a}$ & 190,02 a & 87,21 \\
\hline 105 & $166,99 \mathrm{~b}$ & 299,74 a & 316,66 a & 335,48 a & 58,20 \\
\hline 120 & $220,56 \mathrm{~b}$ & $432,73 \mathrm{a}$ & $471,60 \mathrm{a}$ & 479,86 a & 107,28 \\
\hline 135 & $220,56 \mathrm{~b}$ & $432,72 \mathrm{a}$ & 471,59 a & 479,85 a & 48,61 \\
\hline
\end{tabular}

${ }^{z}$ Valores con la misma letra en cada hilera son estadísticamente iguales (Tukey, $\alpha \leq 0,05$ ). DDT: días después del trasplante; DMS: diferencia mínima significativa.

la extracción de $\mathrm{N}, \mathrm{P}$ y $\mathrm{K}$ es muy lenta en el primer mes de desarrollo del cultivo, pero se incrementa ostensiblemente en el periodo comprendido entre los 40 y 70 días después del trasplante, que coincide con los periodos de floración, amarre y desarrollo del fruto, que de acuerdo con las curvas de crecimiento en términos de acumulación de materia seca (Figura 1), las plantas mostraron tendencia a menos crecimiento a medida que hubo menos suministro nutrimental durante el citado período.

La magnitud más alta de materia seca acumulada en las concentraciones del 75, 125 y $175 \%$ indica que al suministrar más nutrimentos se ven favorecidos los procesos de crecimiento, desarrollo y formación de órganos, de tal forma que se obtuvo un aumento en el rendimiento acumulado por planta, de 
manera diferencial respecto al suministro de nutrimentos (Tabla 4), por la interacción del cultivo con las condiciones de nutrición y ambiente en donde se desarrolló (Schapendonk y Challa 1980; Gifford et al. 1984).

Con la solución al $175 \%$ se obtuvo mayor rendimiento, con diferencias estadísticas significativas respecto a los demás tratamientos. Considerando las distancias de siembra utilizadas, se estimó con dicha solución nutritiva un rendimiento de $273 \mathrm{t} \cdot \mathrm{ha}^{-1}$, que superó en $52 \%$ al rendimiento potencial de 180 t.ha-1. Los valores más bajos se presentaron para la solución al 25\% (Tabla 4), lo que pudo estar relacionado con un alto porcentaje de aborto de flores y frutos.

Tabla 4. Comparación de medias del rendimiento acumulado por planta del cultivo de pepino, a los $120 \mathrm{ddt}$, en cuatro concentraciones de la solución nutritiva de Steiner. Chapingo, México, 2006.

\begin{tabular}{cc}
\hline $\begin{array}{c}\text { Concentración de la solución } \\
\text { nutritiva de Steiner }(\%)\end{array}$ & $\begin{array}{c}\text { Rendimiento } \\
\left.\mathbf{( k g}^{\text {(k) planta }} \mathbf{-}^{-1}\right)\end{array}$ \\
\hline 25 & $3,310 \mathrm{c}^{\mathrm{z}}$ \\
75 & $6,380 \mathrm{bc}$ \\
125 & $6,940 \mathrm{~b}$ \\
175 & $8,200 \mathrm{a}$ \\
DMS & $1,593 \mathrm{~d}$ \\
\hline
\end{tabular}

${ }^{2}$ Valores con la misma letra en la columna son estadísticamente iguales (Tukey, $\alpha \leq 0,05$ ); DMS: diferencia mínima significativa.

\section{CONCLUSIONES}

El rendimiento del cultivo de pepino en invernadero tuvo respuesta al suministro diferencial de nutrimentos, obteniéndose la mayor producción por planta con la solución nutritiva universal de Steiner al 175\% de concentración.

\section{REFERENCIAS}

Alonso, M., Tijerina, L., Sánchez, P., Martínez, A., Aceves, L. y Escalante, J. 2003. Modelo logístico: herramienta para diagnosticar el cuánto y cuándo fertirrigar. Terra Latinoamericana 21(2):225-231.

Barraza, F. 2001. Crecimiento del chile manzano (Capsicum pubescens R. y P.) bajo cuatro soluciones nutritivas en Chapingo, México. Temas Agrarios 6(1112):27-34.

Barraza, F. 2005. Fisiología del Cultivo del Melón. COMPU Gráficas \& Comunicaciones. Universidad de Córdoba. Facultad de Ciencias Agrícolas. Montería, Colombia. 163 p.

Barreiro, M. 1999. El pepino de Sinaloa, calidad y exportación. Claridades Agropecuarias. Secretaría de Agricultura, Ganadería y Desarrollo Rural. México, D.F., p3-16.

Calvo, R., González, J. y Pérez, S. 1994. Manual de Modelos No Lineales en los Ámbitos Agronómico, Ganadero y Forestal. Ministerio de Agricultura, Pesca y Alimentación. Instituto Nacional de Investigación y Tecnología Agraria y Alimentaria. Madrid, España, p110.

Castro, R. y Pérez, M. 2005. El cultivo del pepino en invernadero. Universidad Autónoma Chapingo. Departamento de Fitotecnia. México, p50. 
Challa, H. y Heuvelink, E. 1993. Economic evaluation of crop photosynthesis. Acta Horticulturae 328:219-228.

\section{Charles-Edwards, D., Doley, D. and} Rimmington, G. 1986. Modelling Plant Growth and Development. Academic Press Australia. Australia, p235.

Clavijo, J. 1989. Análisis del crecimiento en malezas. Revista Sociedad Colombiana de Malezas y Fisiología Vegetal, COMALFI 16(12-16):13-16.

De Lint, P.J.A.L. and Heij, G. 1980. Glasshouse cucumber, effects of planting date and night temperature on flowering and fruit development. Acta Horticulturae 118:123-133.

Evans, G. 1972. The Quantitative Analysis of Plant Growth. Blackwell Scientific Publications. Oxford, England, Great Britain, p733.

Fogg, G. 1967. El Crecimiento de las Plantas. Editorial Universitaria de Buenos Aires (EUDEBA). Buenos Aires, Argentina, p327.

Gardner, F., Pearce, R. and Mitchell, R. 1990. Physiology of Crop Plants. Ed. Iowa University Press: AMES. U.S.A., p327.

Gifford, R.M., Thorne, J.H., Hitz, W.D. and Giaquinta, R.T. 1984. Crop Productivity and Photoassimilate Partitioning. Science 225(4644):801-808.

Güenkov, G. 1974. Fundamentos de la Horticultura Cubana. Instituto Cubano del Libro. La Habana, Cuba, p355.
Hunt, R. and Parsons, I. 1974. A computer program for deriving growth-functions in plant growth-analysis. Journal of Applied Ecology 11:297-307.

Hunt, R. 1978. Plant Growth Analysis. Studies in Biology No. 96. The Institute of Biology's. Edward Arnold (Publishers) Limited. London, U.K., p67.

Hunt, R. 1982. Plant growth analysis: second derivatives and compounded second derivatives of splined plant growth curves. Annals of Botany 50(3):317-328.

Hunt, R. 1990. Basic Growth Analysis: Plant Growth Analysis for Beginners. Urwin Hyman Ltd. Londres, Great Britain, p112.

Hyams, D. 2003. Curve Expert 1.3. A comprehensive curve fitting system for Windows $\odot$. Unregistered evaluation copy. USA.

\section{Intagri (Instituto para la innovación tecnológica} en agricultura). 2012. Tercer diplomado internacional en horticultura protegida. www.intagri.com.mx. [18 Marzo 2012].

Jean, R.V. 1984. Mathematical Approach to Pattern and Form in Plant Growth. Wiley-Interscience Publication. New York, U.S.A., p221.

Krug, H. 1985. Growth processes and crop growth modelling. Acta Horticulturae 174:193-204.

Liebig, H. 1980. Physiological and economical aspects of cucumber crop density. Acta Horticulturae 118:149-163. 
Marcelis, L. 1992. Non-destructive measurements and growth analysis of the cucumber fruit. Journal of Horticultural Science 67(4):457-464.

Nátr, L. and Lawlor, D. 2005. Photosynthesis and Plant/Crop productivity and Photosynthetic Products. In: Taylor and Francis Group, LLC (Eds.). Boca Raton, New York, USA., p501-524.

Peil, R. y Galvez, J. 2005. Reparto de materia seca como factor determinante de la producción de las hortalizas de fruto cultivadas en invernadero. R. bras. Agrociência 11(1):5-11.

Pérez, G., F y Martínez L., J. 1994. Introducción a la Fisiología Vegetal. Mundi-Prensa. Madrid, España, p218.

Randolph, A. 1999. Tips de producción intensiva. Análisis de los diferentes sistemas de producción. Revista Productores de Hortalizas. Año 8, No.4. Abril 1999. Meister Publishing Co. D.F., México, p16-18.

Rojas, P., Pérez, M., Colinas, M., Sahagún, J. y Avitia, J. 2008. Modelos matemáticos para estimar el crecimiento del fruto de chile manzano (Capsicum pubescens R. y P.). Revista Chapingo. Serie horticultura 14(3):289-294.

Salisbury, B. F. y Ross, C. W. 1994. Fisiología Vegetal. Grupo Editorial Iberoamérica S.A. de C.V. D.F., México, p759.
SAS Institute Inc. 2002. Statistical Analysis System. The $\mathrm{SAS}^{\odot}$ system 9 for Windows ${ }^{\odot}$ The Power to Know. Cary, NC, 27513, USA.

Schapendonk, A. and Challa, H. 1980. Assimilate requirements for growth and maintenance of the cucumber fruit. Acta Horticulturae 118:73-82.

Steiner, A. 1961. A universal method for preparing nutrient solutions of a certain desired composition. Horticultural Experiment Station, Naaldwijk, Netherlands. Reprint from Plant and Soil. XV(1961):134-154.

Skierkowski, J.; Dobrzanska, J. 1980. Greenhouse cucumber production and research in Poland. Acta Horticulturae 118: 41-55.

Tanaka, A. y Yamaguchi, J. 1984. Producción de Materia Seca, Componentes del Rendimiento y Rendimiento del grano en Maíz. Traducido al español de J. Kohashi Shibata. Taller del Colegio de postgraduados. Montecillo, México, p120.

Valadez, A. 1998. Producción de Hortalizas. Editorial UTEHA, D.F., México, p298.

Visser, A. J. 1980. Economic aspects of cucumber growing in the Netherlands. Acta Horticulturae 118: 11-16.

Wild, A. 1992. Condiciones del Suelo y Desarrollo de las Plantas Según Russel. Mundi-Prensa. Madrid, España, 1045p. 